Applied Mathematical Sciences, Vol. 4, 2010, no. 60, 2993 - 2998

\title{
Effect of Pollution Tax on Population in a Polluted Environment
}

\author{
Jiwei He \\ School of Mathematics and Physics \\ North China Electric Power University \\ Baoding, 071003, P. R. China \\ hejiwei313@163.com
}

\begin{abstract}
This paper establishes a mathematical model to study the effect of pollution tax on the long behavior of the population living in a polluted environment. We give the sufficient condition for persistence of the population under some conditions, and give some numerical simulation.
\end{abstract}

Mathematics Subject Classification: 34C34; 92D25

Keywords: Pollution tax; Environmental pollution; Persistence

\section{Introduction}

Due to the rapid development of industrialization, presence of toxicant in the environment decreases the growth rate of species and its carrying capacity. It is well known that the pollution of environment is a very serious problem in the world today. The study of the effect of toxicant on population is quite important. One efficient way to control the environment pollution is imposing pollution tax on toxicant emitters. In this paper, we introduce a mathematical model to analyze this problem.

The study of deterministic dynamic population models with toxicant effect was proposed by Hallam and his colleagues in 1980s[1,2,3]. This model has been improved by many scholars[4,5]. He Zerong et al in [6] studied the survival of a single logistic species in a polluted environment, considering the organism's uptake of toxicant from the environment and egestion of toxicant into the environment. In [7], Ma et al studied a Leslie resource-consumer model, and obtained the threshold between the persistence and extinction of the consumer. In [8], the authors improved the results of [7]. In [9], Buonomo et al studied the effect of variation of the population on the toxicant concentration in the 
organism and environment. In [10] and [11], we gave some concrete analysis of the ODE system in [9], and improved the results into Gallopin resourceconsumer system.

In this paper, we suppose that pollution tax is imposed on toxicant emitters if their emission crosses the permissible limit, limit up to which there is no harm to the population. In this paper, we assume that all the emission cross the permissible limit. What's more, we also consider the negative effect of tax evasion. We obtain the sufficient condition for persistence of the population, and give some numerical simulation.

\section{The Model}

Our study relies on the hypothesis of a complete spatial homogeneous environment, and no migration. Let $x(t)$ represent the population of the consumer at time $t, C_{e}(t)$ be the toxicant concentration of the environment at time $t$, $C_{0}(t)$ be the toxicant concentration in a body, and $I(t)$ be the environmental tax imposed on the emitters. We assume that the living organisms absorb part of this toxicant into their bodies so that the dynamics of the population is affected by this (internal) toxicant. We will study their relationship, and give some sufficient conditions gurantee the persistence of the population in a polluted environment. The single species model in a polluted environment is given as follows:

$$
\begin{aligned}
\dot{x}(t) & =x(t)\left(r_{0}-\alpha C_{0}(t)-f x(t)\right), \\
\dot{C}_{0}(t) & =k C_{e}(t)-(g+m) C_{0}(t), \\
\dot{C}_{e}(t) & =-k C_{e}(t) x(t)+g x(t) C_{0}(t)-h C_{e}(t)+Q-\rho I(t), \\
\dot{I}(t) & =\theta\left(C_{e}(t)-A\right)-\theta_{0} I(t) .
\end{aligned}
$$

The initial values $x(0)=x_{0}>0, C_{0}(0) \geq 0, C_{e}(0) \geq 0, I(0) \geq 0$. All the coefficients in the model are positive. $r_{0}$ is the intrinsic growth rate in absence of toxicant, $\alpha$ is the response of population to the toxicant, $f$ is a positive constant representing intra specific competition of the population. $k$ represents the uptake rate of the toxicant from the environment by the population. $g$ is the egestion rate of toxicant input to the environment from the population. $m$ represents metabolism rate of the toxicant. $h$ denotes the losses rate of the toxicant in the environment due to natural degradation. $Q$ represents the exogenous rate of toxicant input into the environment beyond the initial toxicant. $\rho$ is the tax repulsion coefficient. Tax is imposed only if $C_{e}$ crosses the permissible limit $A$ (limit up to which there is no harm to the population). $\theta$ and $\theta_{0}$ are constants, $\theta_{0}$ denotes some practical difficulties on implementing the fool proof tax system.

\section{$3 \quad$ Main Results}


Lemma 1 If $h>k, g+m-g r_{0} / f>0$, then the region $\Omega=\left\{\left(x(t), C_{0}(t), C_{e}(t), I(t)\right)\right.$ : $\left.0 \leq x(t) \leq r_{0} / f, 0 \leq C_{0}(t)+C_{e}(t) \leq Q / \delta, 0 \leq I \leq \frac{\theta Q}{\theta_{0} \delta}\right\}$ is the invarial set of model $\left(M_{1}\right)$ here $\delta=\min \left\{h-k, g+m-g r_{0} / f\right\}$.

Proof: $x=0$ is the solution of equation (1) obviously. If $x_{0}>0$, then $x(t)>0, t \in R^{+}$. From

$\left.\dot{C}_{0}(t)\right|_{C_{0}=0, C_{e}>0}>0,\left.\quad \dot{C}_{e}(t)\right|_{C_{e}=0, C_{0}>0}=g x(t) C_{0}(t)+Q-\rho I(t)>0, \dot{I}(t)_{I=0, C_{e}(t)>A}>0$,

we have

$$
x(t) \geq 0, \quad C_{e}(t) \geq 0, \quad I(t) \geq 0 .
$$

From equation (1) we get

$$
\dot{x}(t) \leq x(t)\left(r_{0}-f x(t)\right),
$$

the comparison theorem gives

$$
\limsup _{t \rightarrow \infty} x(t) \leq r_{0} / f
$$

Equations (2) and(3) give that

$$
\begin{aligned}
\dot{C}_{0}(t)+\dot{C}_{e}(t) & \leq Q+k C_{e}(t)-g C_{0}(t)-m C_{0}(t)-h C_{e}(t)+g x(t) C_{0}(t) \\
& \leq Q-\left(g+m-\frac{g r_{0}}{f}\right) C_{0}(t)-(h-k) C_{e}(t) \\
& <Q-\delta\left(C_{0}(t)+C_{e}(t)\right) .
\end{aligned}
$$

Then we have

$$
\limsup _{t \rightarrow \infty}\left(C_{0}(t)+C_{e}(t)\right) \leq \frac{Q}{\delta}
$$

From equation (4) we have $\dot{I}(t) \leq \theta C_{e}(t)-\theta_{0} I \leq \theta \frac{Q}{\delta}-\theta_{0} I(t)$. Using the comparison theorem again, we can get

$$
\limsup _{t \rightarrow \infty} I(t) \leq \frac{\theta Q}{\theta_{0} \delta}
$$

From $(5),(6),(7),(8)$ we get $\Omega$ is the invariant set of model $\left(M_{1}\right)$.

Theorem 1 If $\delta>\max \left\{\frac{\rho \theta}{\theta_{0}}, \frac{\alpha Q}{r_{0}}\right\}, \frac{Q\left(1-\frac{\rho \theta}{\delta \theta_{0}}\right)}{h+k r_{0} / f}>A$, then model $\left(M_{1}\right)$ is persistent uniformly.

Proof. From equation (1) we have $\dot{x}(t) \geq x(t)\left(r_{0}-\alpha Q / \delta-f x\right)$, then

$$
\liminf _{t \rightarrow \infty} x(t) \geq\left(r_{0}-\alpha Q / \delta\right) / f
$$


Equation (3) gives that

$$
\dot{C}_{e}(t) \geq Q-\rho \frac{Q \theta}{\delta \theta_{0}}-\frac{k r_{0}}{f} C_{e}(t)-h C_{e}(t)=Q\left(1-\frac{\rho \theta}{\delta \theta_{0}}\right)-\left(h+\frac{k r_{0}}{f}\right) C_{e}(t)
$$

Using the comparison theorem, we can get

$$
\liminf _{t \rightarrow \infty} C_{e}(t) \geq Q\left(1-\frac{\rho \theta}{\delta \theta_{0}}\right) /\left(h+\frac{k r_{0}}{f}\right) .
$$

From equation (2) we get

$$
\dot{C}_{0}(t) \geq k Q\left(1-\frac{\rho \theta}{\delta \theta_{0}}\right) /\left(h+k r_{0} / f\right)-(g+m) C_{0}(t),
$$

then

$$
\liminf _{t \rightarrow \infty} C_{0}(t) \geq \frac{k Q\left(1-\frac{\rho \theta}{\delta \theta_{0}}\right)}{(g+m)\left(h+k r_{0} / f\right)} .
$$

Equation (4) implies that

$$
\dot{I}(t) \geq \theta\left(\frac{Q\left(1-\frac{\rho \theta}{\delta \theta_{0}}\right)}{h+k r_{0} / f}-A\right)-\theta_{0} I(t),
$$

then we have

$$
\liminf _{t \rightarrow \infty} I(t) \geq \frac{\theta}{\theta_{0}}\left(\frac{Q\left(1-\frac{\rho \theta}{\delta \theta_{0}}\right)}{h+k r_{0} / f}-A\right) .
$$

And then from (9), (10), (11), (12) and Lemma 1, we get

$$
\begin{gathered}
\left(r_{0}-\alpha Q / \delta\right) / f \leq \liminf _{t \rightarrow \infty} x(t) \leq \limsup _{t \rightarrow \infty} x(t) \leq r_{0} / f, \\
\frac{k Q\left(1-\frac{\rho \theta}{\delta \theta_{0}}\right)}{(g+m)\left(h+k r_{0} / f\right)} \leq \liminf _{t \rightarrow \infty} C_{0}(t) \leq \limsup _{t \rightarrow \infty} C_{0}(t) \leq \frac{Q}{\delta} \\
Q\left(1-\frac{\rho \theta}{\delta \theta_{0}}\right) /\left(h+\frac{k r_{0}}{f}\right) \leq \liminf _{t \rightarrow \infty} C_{e}(t) \leq \limsup _{t \rightarrow \infty} C_{e}(t) \leq \frac{Q}{\delta} \\
\frac{\theta}{\theta_{0}}\left(\frac{Q\left(1-\frac{\rho \theta}{\delta \theta_{0}}\right)}{h+k r_{0} / f}-A\right) \leq \liminf _{t \rightarrow \infty} I(t) \leq \limsup _{t \rightarrow \infty} I(t) \leq \frac{\theta Q}{\theta_{0} \delta}
\end{gathered}
$$

Then from $(13)-(16)$ we know that $\left(M_{1}\right)$ is persistent uniformly. 


\section{Numerical Simulation}

In this section, we give some examples. Set $r_{0}=1, \alpha=1, f=0.5, k=$ $1, g=1, m=2, h=1, \rho=0.5, \theta=2, \theta_{0}=0.5, A=0.2$, and the initial values are $x(0)=0.5, C_{0}(0)=0.1, C_{e}(0)=0.2, I(0)=0$, then the numerical solution of model $\left(M_{1}\right)$ is as following figure 1. If there is no environmental tax, the solution of model $\left(M_{1}\right)$ is illustrated in figure 2 .

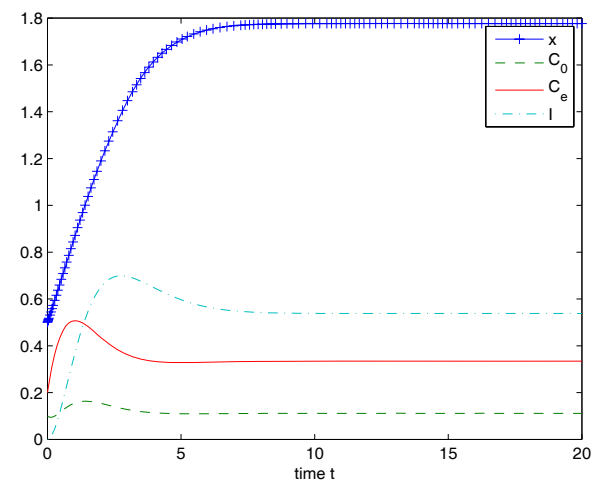

Fig. $1 . Q=1$

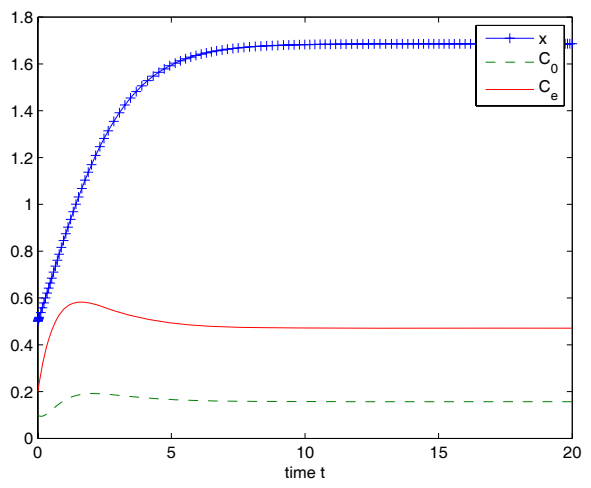

Fig. 2. $Q=1, \rho=0$

From these plots, we can see that the toxicant concentration of environment increases to 0.5 from 0.3 and the amount of the population decreases to 1.7 from 1.8 .

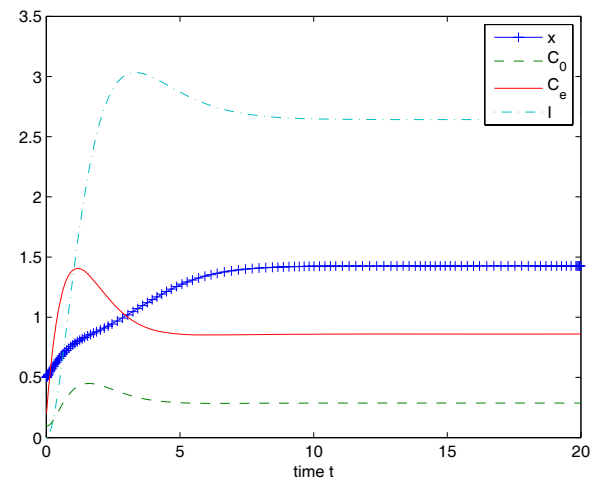

Fig. 3. $Q=3$

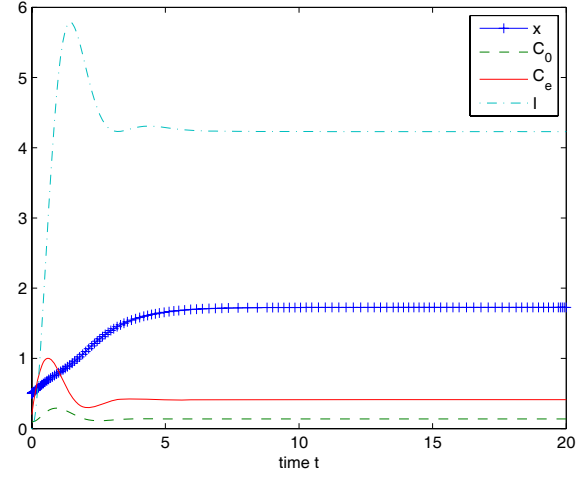

Fig. 4. $Q=3, \theta=10$

In fig. 1 and fig. 3 , we can find that when $Q$ increases from 1 to 3 , which will lead the increase the tax and sharp decrease of the amount of the population. But when the heavy $\operatorname{tax}((\theta=10))$ is imposed on the emitters, the balanced amount of the population will recover to 1.8 quickly. $\theta$ is the key parameter which need to choose very carefully in order to maintain the equilibrium level of population. 


\section{References}

[1] T. G. Hallam, C. E. Clark, R. R. Lassider, Effects of toxicant on population, A qualitative approach I: Equilibrium environmental exposure, Ecological Modelling, 8(1983), 291-304.

[2] T. G. Hallam, C. E. Clark, G. S.Jordan, Effects of toxicant on population, A qualitative approach II: First Order Kinifics, Journal of Mathematical Biology, 18(1983), 25-37.

[3] T. G. Hallam, J. T. Deluna, Effects of toxicant on population, A qualitative approach III: Environmental and food chain pathways, Journal of Theoretical Biology, 109(1984), 411-429.

[4] Z. Ma, The Study of Biology Model, Hefei, Anhui Education Press1996.

[5] L. Chen, Mathematical Models and Methods in Ecology, Chendu, Sichuan Science Press, 2003.

[6] Z. He, Z. Ma, The effect of toxicant and harvest on the logistic model, Journal of Biomathematics, 12(1997), 230-237.

[7] Z. Ma, B. Song, T. G. Hallam, The threshold between persistence and extinction of a population in a polluted environment, Bulletin of Mathematical Biology, 51(1989), 311-323.

[8] Z. He, Z. Ma, On the effects of pollution and catch to a logistic population, Journal of Biomathematics, 14(1999), 281-287.

[9] B. Buonomo, A. D. Liddo, I. Sgura, A diffusive-convective model for the dynamics of population-toxicant intentions: some analytical and numerical results, Mathematical Biosciences, 157(1999), 37-64.

[10]J. He, K. Wang, The survival analysis for a population in a polluted environment. Nonlinear Analysis: Real World Applications, 3(2009), 1555-1571.

[11]J. He, K. Wang, The survival analysis for a single-species population model in a polluted environment, Applied Mathematical Modelling, 31(2007), 2227-2238.

Received: May, 2010 